\title{
INNOVACIÓN EN DOCENCIA: METODOLOGÍA DE “APRENDIZAJE Y SERVICIO" EN CONTABILIDAD DE COSTOS
}

\section{INNOVATION IN TEACHING, METHODOLOGY “SERVICE LEARNING" ON COST ACCOUNTING}

\author{
Verónica Pizarro Torres ${ }^{1}$, Beatriz Hasbun Held ${ }^{2}$ y Tomás González Cifuentes ${ }^{3}$
}

\section{RESUMEN}

La Educación Superior en Chile está enfrentando cada día mayores desafíos asociados a la calidad de sus programas, su capacidad de adaptación a nuevos contenidos y demandas de la sociedad y el mercado, la permanente competencia a nivel nacional e internacional en términos de la captación de matrículas, y el desarrollo de las competencias disciplinares y genéricas con las cuales egresan sus estudiantes. Este último desafío se relaciona estrechamente con la innovación en los procesos y metodologías de enseñanza, y la vinculación que establezca la universidad con su entorno.

Es posible señalar que una de las formas, estudiadas a nivel nacional e internacional, de enlazar el proceso de formación profesional con las necesidades del entorno (sociedad) es la implementación de la metodología de Aprendizaje y Servicio, (Service Learning, A+S). El presente trabajo pretende analizar y evaluar la experiencia que esta metodología ha tenido en el curso de Fundamentos de Costos de la Facultad de Economía y Negocios de la Universidad de Chile.

Entre los principales resultados que serán presentados se destaca la adecuación del cuestionario para caracterizar a los estudiantes que participaron en la metodología en el contexto de la asignatura, el alto nivel de motivación social manifestado por los estudiantes, el potencial de la metodología para fomentar el desarrollo de competencias genéricas, y la efectividad de la metodología A+S para reforzar los contenidos de la asignatura.

Palabras claves: Aprendizaje y Servicio, Fundamento de Costos, Innovación.

Recepción: 26/01/2016. Aprobación: 4/04//2016.

\begin{abstract}
Nowadays, Higher Education in Chile faces major challenges related to the quality of its programs, its ability to adapt to new content and demands of society and the market competition at a national and international level in terms of recruitment and enrollment of students, and the disciplinary and generic skills with which students graduate. The latter is closely related to innovation processes and teaching methodologies and the relationship with the external environment of the university..

One of the ways, studied at international and national level, to link the formation process with the needs of the environment (society) is the implementation of the Service Learning methodology (S+L). This paper analyzes the implications that this methodology has had on the Cost Foundation course

1 Departamento Control de Gestión y de Sistemas de Información, Facultad de Economía y Negocios, Universidad de Chile. Santiago, Chile. vpizarro@ fen.uchile.cl

2 Centro de Enseñanza y Aprendizaje, Facultad de Economía y Negocios, Universidad de Chile. Santiago, Chile. bhasbun@fen.uchile.cl

3 Centro de Desarrollo Docente, Pontificia Universidad Católica de Chile. Santiago, Chile. tomas.gonzalez@uc.cl
\end{abstract}


of the Economics and Business Faculty of at the University of Chile

The main results to be presented highlights the adequacy of the questionnaire to characterize the students who participated in the methodology in the context of the subject, the high level of social motivation expressed by students, the potential of the methodology to foster the development of generic skills, and ultimately the effectiveness of the S+L methodology to reinforce the contents of the subject.

Keywords: Service Learning, Cost Foundation, Innovation.

\section{INTRODUCCIÓN}

En la actualidad, la Educación Superior en Chile enfrenta diversos retos, entre los cuales destaca la necesidad de situarse en un contexto de gran competitividad nacional e internacional en términos de calidad y capacidad de adecuar los procesos de formación, junto a la expectativa de que las instituciones de Educación Superior se posicionen como motor de desarrollo local de su entorno (Zabalza, 2002).

Avanzar en dicha visión implica, desde el enfoque de la Responsabilidad Social Universitaria, ampliar la manera en que las instituciones y programas de estudio se vinculan con el entorno más allá de actividades de extensión que se realizan con la comunidad. En ese sentido, es posible abordar la vinculación con el medio desde la formación, lo que permite potenciar la concepción ética, social y sobre el futuro rol profesional del estudiantado; mejorar el aprendizaje (Puig et al., 2007); y, contribuir al desarrollo local mediante la transferencia de conocimientos.

La Universidad de Chile, en los últimos años, ha experimentado un profundo proceso de modernización de su formación de pregrado. En esta nueva etapa, el foco del proceso de formación se ha trasladado del docente al estudiante, lo que genera una atención particular por el desarrollo de competencias y metodologías innovadoras e inclusivas, que fomenten el desarrollo de profesionales integrales preparados para enfrentar los desafíos actuales de su área. La Facultad de Economía y Negocios (FEN) de la Universidad de Chile, ha buscado responder a este propósito a través de la implementación de metodologías activas que vinculen los objetivos de aprendizaje de sus asignaturas con el contexto real. Una alternativa para lograr tal propósito es el Aprendizaje y Servicio $(\mathrm{A}+\mathrm{S})$, definido teóricamente como una metodología asociada a un curso obligatorio, en la cual los estudiantes participan en una actividad de servicio organizada que busca dar respuesta a necesidades de la comunidad, reflexionando de forma tal que promueve en el estudiante una comprensión de su disciplina que va más allá del contenido específico del curso, y entregando un sentido avanzado de responsabilidad social (Bringle \& Hatcher, 1995; Furco,1998; Puig et al., 2007; Puig 2009; \& Tapia, 2010).

En términos de la formación profesional en el área de contabilidad, el curso Fundamentos de Costos impartido para las carreras de Contador Auditor e Ingeniería en Sistemas de Información y Control de Gestión, ha implementado desde el 2008 la metodología A+S, vinculando los resultados de aprendizaje del curso con las necesidades de emprendedores locales.

El objetivo de este trabajo es presentar los resultados de la experiencia en la implementación de la metodología $\mathrm{A}+\mathrm{S}$ en el curso de Fundamentos de Costos en el año 2014, la cual fue evaluada a través de un cuestionario diseñado y validado por el Centro de Enseñanza y Aprendizaje de la Facultad de Economía y Negocios de la Universidad de Chile. Para el diseño del cuestionario fueron considerados tanto los constructos teóricos que sustentan la metodología, como la definición de Responsabilidad Social, y está compuesto por las dimensiones de motivación, compromiso ciudadano, desarrollo de competencias genéricas y satisfacción de los estudiantes con la metodología.

\section{APRENDIZAJE Y SERVICIO EN LA CONTABILIDAD}

Existen experiencias y reflexiones sistematizadas en cuanto a la implementación de la metodología de $\mathrm{A}+\mathrm{S}$ en áreas asociadas a la Economía y los Negocios. En ese sentido, Zlotkowski (1996) postula que una de las mayores críticas realizadas a la formación contemporánea en la formación en negocios, es su incapacidad de desarrollar en los estudiantes comportamientos éticos y una sensibilidad respecto al entorno ex- 
terno a la empresa. En ese sentido, señala que la metodología de Aprendizaje y Servicio otorga a las Escuelas de Negocio una excelente oportunidad para suplir dicha deficiencia.

Rama (1998), al estudiar el Aprendizaje y Servicio contextualizado al área de contabilidad, afirma que la actividad debe estar diseñada de tal forma que: a) recoja las necesidades de la comunidad, b) mejore la comprensión del contenido de los cursos de contabilidad, c) ayude a desarrollar una comprensión más amplia de la profesión del contador, d) aporte a promover el sentido de responsabilidad cívica e) desarrolle las habilidades de trabajo en equipo, comunicación y habilidades interpersonales.

Una experiencia destacada ha sido la de Bentley College, en la cual un $25 \%$ de los académicos integraron actividades de Aprendizaje y Servicio a sus cursos. Entre los principales resultados señalan: la elaboración de productos y servicios que permitieron que los estudiantes desarrollaran sus conocimientos al mismo tiempo que beneficiaran a las agencias con las cuales trabajaron. Asimismo, los estudiantes reportaron un aumento de sensibilidad frente a temáticas de responsabilidad social y justica, conectando su labor profesional con la de ser ciudadano (Kenworthy,; 1996).

Por otra parte, el Daniel College of Business, de la Universidad de Denver (Wittmer, 2004) sistematizó la experiencia de implementación durante 8 años de un curso de Master in Business Administration (MBA). La percepción de los estudiantes, evaluada a través de una encuesta, en su mayoría manifestaron un aumento en el reconocimiento de las necesidades del entorno y mayor conciencia de compromiso.

Por otro lado, Flannery y Pragman (2008) evaluaron el compromiso ciudadano y responsabilidad social, aplicación de habilidades y desarrollo profesional, asociado a la implementación de la Metodología de A+S en la formación en administración. En general, el estudio demuestra que al año de implementación de la metodología en un curso, las percepciones de los estudiantes mejoran significativamente. Asimismo, los estudiantes manifestaron que la experiencia les aportó a sentirse comprometidos cívicamente, a la vez que reconocen la actividad como una aplicación profesional real.

Por último, Zamora (2012) sistematiza su ex- periencia de aplicación de $\mathrm{A}+\mathrm{S}$ durante tres años en un curso introductorio de contabilidad. Dentro de los resultados destaca que los estudiantes manifiestan que la actividad de vinculación les aportó a mejorar su aprendizaje en aspectos específicos de conceptos de contabilidad, por lo que evaluaban la experiencia como positiva para su aprendizaje.

\section{APRENDIZAJE Y SERVICIOS EN EL CURSO DE FUNDAMENTOS DE COSTOS}

La carrera de Contador Auditor de la Facultad de Economía y Negocios (FEN) de la Universidad de Chile comienza en 2008 un proceso de evaluación y rediseño de su plan de estudios. Esta reforma tiene como objetivo principal centrar la formación en el estudiante, además de transitar un modelo basado en contenidos hacia uno basado en competencias. En el año 2009, dentro del proceso de adaptación del syllabus del curso de Fundamentos de Costos, se considera por primera vez la incorporación de la metodología de Aprendizaje y Servicio $(\mathrm{A}+\mathrm{S})$, como medio tanto para desarrollar las competencias específicas asociadas al dominio de contabilidad, como para potenciar la competencia genérica de responsabilidad social y compromiso ciudadano. En específico, busca que los estudiantes adquieran conocimientos relacionados con contabilidad de costos y su rol como herramienta de apoyo a la dirección de empresas, distinguiendo claramente los métodos de costeo y su aplicación a los sistemas de costos y, a su vez, que los puedan poner en práctica con un trabajo en conjunto con pequeñas y medianas empresas que no tengan claro los costos de sus productos y/o servicios que ofrecen. De esta manera, la aproximación del estudiante hacia los contenidos del curso ocurre de manera situada, contemplando los desafíos reales del mercado y las implicancias sociales y personales asociadas a los socios comunitarios. Lo anterior permite que el estudiante integre los aprendizajes disciplinarios, desarrollando a su vez, la competencia de responsabilidad social y compromiso ciudadano.

En la siguiente tabla se muestran las principales características de Fundamentos de Costos. 
Tabla I: Cuadro de características de Fundamentos de Costos.

\begin{tabular}{|c|l|}
\hline Temática & \multicolumn{1}{c|}{ Características } \\
\hline Semestre & $4^{\circ}$ a $5^{\circ}$ semestre/Ciclo de Formación Básico \\
\hline Intención formativa & Exponer a los estudiantes a una problemática empresarial real. \\
\hline $\begin{array}{c}\text { Competencia Genérica } \\
\text { Principal }\end{array}$ & Responsabilidad Social y Compromiso Ciudadano. \\
\hline $\begin{array}{c}\text { Competencias } \\
\text { Específicas }\end{array}$ & $\begin{array}{l}\text { Analizar elementos, modelos, métodos, criterios y sistemas de costos. } \\
\text { Elaborar y proporcionar información contable y de gestión orientada a } \\
\text { la toma de decisiones con el propósito de apoyar la toma de decisiones. }\end{array}$ \\
\hline Tipo de empresa & $\begin{array}{l}\text { Micro, Pequeñas, y medianas empresas, con fines de Lucro, preferente } \\
\text { productivas. }\end{array}$ \\
\hline $\begin{array}{c}\text { Planteamiento del } \\
\text { Desafío o Problema }\end{array}$ & $\begin{array}{l}\text { Problema específico de Identificación, descripción, tipificación de los } \\
\text { diversos costos de la empresa. Determinación de los costos de los pro- } \\
\text { ductos y servicios. Finalmente la detección de problemas de Costos y } \\
\text { sus alternativas de solución }\end{array}$ \\
\hline
\end{tabular}

\section{FASES DE IMPLEMENTACION DE LA METODOLOGIA A+S}

Basándose el los postulados de Tapia (2007), la implementación de $\mathrm{A}+\mathrm{S}$ en el curso de Fundamento de Costos se ha realizado a partir de tres grandes fases:

Fase 1: Reclutamiento y selección de socios comunitarios

Esta fase es anterior al comienzo de la ejecución del curso. En específico, se centra en el proceso de búsqueda y vinculación con las empresas que trabajarán como socios comunitarios, identificando sus requerimientos de asesoría, la coherencia de estas necesidades con las asignaturas, y las expectativas de ambas partes respecto al trabajo conjunto.

\section{Fase 2: Implementación de la metodología}

La segunda fase se centra en el trabajo entre los socios comunitarios seleccionados, el equipo docente y los estudiantes, y considera: a) la presentación del curso a los estudiantes y contacto inicial con Socios Comunitarios, b) el proceso de inmersión de los actores y desarrollo del proyecto en conjunto, c) la entrega de un informe final al socio ecomunitario con los resultados de la asesoría, y d) la generación de capacidades en los emprendedores mediante talleres de capacitación.

\section{Fase 3: Monitoreo y Evaluación}

La tercera fase, corresponde a una fase transversal durante el desarrollo del curso, y contempla tanto hitos de reflexión conjunta de estudiantes y socios comunitarios durante el semestre, con el objeto de gestionar de manera eficiente y mejorar continuamente esta metodología en pos de mejorar los resultados de aprendizaje de los estudiantes y de satisfacción de los empresarios, como la aplicación de encuestas para la evaluación de la metodología.

\section{METODOLOGÍA DE ESTE ESTUDIO}

El presente estudio es de tipo cuantitativo, descriptivo, en cuanto busca caracterizar la percepción de los estudiantes participantes de un curso con metodología $\mathrm{A}+\mathrm{S}$, sobre sus motivaciones, experiencias y percepción de desarrollo de competencias y comportamientos cívicos. Para ello, se ha utilizado un cuestionario diseñado y validado por el Centro de Enseñanza y Aprendizaje de la Facultad de Economía y Negocios de la Universidad de Chile.

\section{Población y Muestra}

La población del curso corresponde a 73 estudiantes, de los cuales participaron 37, lo que corresponde a un $90 \%$ de confianza y un $10 \%$ de error muestral. 


\section{Levantamiento y Análisis de Datos}

La aplicación del instrumento se realizó a partir de una muestra cautiva en el curso de Fundamentos de Costos a finales del semestre. La encuesta se aplicó en papel como auto encuesta, los estudiantes respondían de forma individual teniendo que firmar los respectivos consentimientos informados.

Se realizó un análisis estadístico descriptivo, mediante el software Excel, que contempla el cálculo de medias y de frecuencias relativas de las categorías de respuesta del instrumento.

\section{RESULTADOS PERCEPCIÓN ESTUDIANTES}

A continuación se presentan los resultados de la aplicación del instrumento “Cuestionario
Evaluación Aprendizaje Vinculado al Medio", aplicado al curso Fundamentos de Costos en la Facultad de Economía de la Universidad de Chile en el periodo Otoño 2014.

\section{Dimensión de Caracterización de Estudiantes según Motivación}

En cuanto a las motivaciones personales, "Ser un profesional que aporte a la construcción de una sociedad justa, solidaria y sustentable" junto con "Tener conciencia social" son las aseveraciones que obtuvieron el promedio más alto $(6,5)$, en una escala del 1 a 7 . Por otra parte, finalizar la carrera con reconocimiento por parte de la Universidad es el promedio más bajo $(5,7)$.

Tabla II. Motivaciones Personales.

\begin{tabular}{|l|c|}
\hline \multicolumn{1}{|c|}{ Motivación personal } & Promedio \\
\hline $\begin{array}{l}\text { Ser un profesional que aporte a la construcción de una sociedad justa, solidaria y } \\
\text { sustentable }\end{array}$ & 6,5 \\
\hline Tener conciencia social & 6,5 \\
\hline Comprender los problemas sociales que enfrenta actualmente el país & 6,3 \\
\hline $\begin{array}{l}\text { Comprometerse con acciones que la comunidad reconoce como relevantes para su } \\
\text { desarrollo }\end{array}$ & 6,1 \\
\hline Promedio asignatura & 6,1 \\
\hline Continuar estudios de postítulo y/o especialización & 5,9 \\
\hline Finalizar la carrera con reconocimiento por parte de la Universidad & 5,0 \\
\hline
\end{tabular}

Por otra parte, se solicitó a los estudiantes que seleccionaran tres orientaciones hacia las cuales consideraran que se dirigían sus acciones. Las opciones corresponden a orientaciones o intereses personales que pudieran tener los estudiantes, como lo ejemplifica la categoría "Crecer personalmente" $(89,2 \%)$. Como se evidencia, la tendencia indica que los estudiantes tienen menor orientación a "Superar sus prejuicios" $(10,8 \%)$ y "Obtener reconocimiento" (10,8\%). 
Tabla III. Orientaciones personales.

\begin{tabular}{|l|c|c|}
\hline \multicolumn{1}{|c|}{ Orientaciones personales } & Frecuencia & $\%$ \\
\hline Crecer personalmente & 33 & 89,2 \\
\hline Enfrentar desafíos & 28 & 75,7 \\
\hline Ayudar a los demás & 28 & 75,7 \\
\hline Conocer nuevas perspectivas y/o visiones & 18 & 48,6 \\
\hline Obtener reconocimiento & 6 & 16,2 \\
\hline Superar tus prejuicios & 4 & 10,8 \\
\hline
\end{tabular}

En términos de los objetivos que tienen los estudiantes al cursar la Asignatura de Fundamentos de Costos, los más elegidos fueron "Acercarse al mundo laboral" (51,4\%); "Alcanzar los resultados de aprendizaje de la asignatura" y "Conocer los con- tenidos y su aplicabilidad" (48,6\%). Los objetivos menos elegidos por otra parte fueron "Lograr los objetivos de la asignatura" $(10,8 \%)$ y "ampliar mis perspectivas profesionales" $(18,9 \%)$.

Tabla IV. Objetivos de los estudiantes sobre la asignatura Fundamento de Costos.

\begin{tabular}{|l|c|c|}
\hline Objetivos estudiantes sobre la asignatura & Frecuencia & $\%$ \\
\hline Acercarme al mundo laboral & 19 & 51,4 \\
\hline Alcanzar los resultados de aprendizaje de la asignatura & 18 & 48,6 \\
\hline Conocer los contenidos y su aplicabilidad & 18 & 48,6 \\
\hline Desarrollar las competencias de la asignatura & 14 & 37,8 \\
\hline Obtener buenas calificaciones & 14 & 37,8 \\
\hline Integrar los conocimientos previos & 11 & 29,7 \\
\hline Capacitarme profesionalmente & 11 & 29,7 \\
\hline Ampliar mis perspectivas profesionales & 7 & 18,9 \\
\hline Lograr los objetivos de la asignatura & 4 & 10,8 \\
\hline
\end{tabular}

\section{DIMENSIÓN IMPLEMENTACIÓN $\mathrm{DE} A+\mathrm{S}$}

Se consultó a los estudiantes acerca de su relación con los diferentes participantes de la metodología de vinculación con el medio, acerca de su evaluación del desempeño y compromiso de los mismos y la comunicación que tuvieron con ellos. Para ello, los estudiantes debían asignar a cada uno de ellos en las diferentes instancias. El detalle de las evaluaciones se puede observar en la Tabla 5 . 
Tabla V. Relación y Desempeño entre los participantes.

\begin{tabular}{|l|c|c|c|}
\hline \multicolumn{1}{|c|}{ Participante } & Relación & Desempeño y compromiso & Comunicación \\
\hline Grupo de trabajo & 6,4 & 5,9 & 6,3 \\
\hline Socio Comunitario & 5,9 & 6,0 & 6,0 \\
\hline Docente & 5,5 & 6,2 & 6,0 \\
\hline Compañeros de curso & 6,0 & 5,8 & 5,8 \\
\hline Ayudantes & 5,8 & 6,0 & 5,8 \\
\hline Promedio & 5,9 & 5,9 & 5,9 \\
\hline
\end{tabular}

Respecto a la relación con los distintos participantes de la asignatura, el grupo de trabajo es el cual obtiene la más alta calificación, respecto al desempeño y compromiso la nota más alta la obtiene el docente mientras que el grupo de trabajo obtiene nuevamente la calificación más alta respecto a la comunicación.

En relación al desarrollo de la metodología, se consultó a los estudiantes acerca de ciertas afirmaciones ante las cuales manifestar su nivel de acuerdo en relación a la implementación de la metodología. Los resultados son los siguientes:

Tabla VI. Satisfacción con la Metodología A+S.

\begin{tabular}{|l|c|c|}
\hline \multicolumn{1}{|c|}{ Afirmaciones } & Frecuencia & $\%$ \\
\hline $\begin{array}{l}\text { La actividad de vinculación con el medio es adecuada para el nivel de conoci- } \\
\text { mientos, competencias y destrezas que tengo }\end{array}$ & 36 & 97,3 \\
\hline Los aprendizajes del curso fueron significativos para mí & 36 & 97,3 \\
\hline Después de esta actividad me siento más capacitado laboralmente & 36 & 97,3 \\
\hline La actividad de vinculación con el medio reforzó los contenidos de la asignatura & 34 & 91,9 \\
\hline La asesoría que brindé al socio comunitario es viable de aplicar & 34 & 91,9 \\
\hline Me gustaría volver a desarrollar una actividad como esta en otras asignaturas & 33 & 89,2 \\
\hline $\begin{array}{l}\text { El tipo de asesoría planificada en la asignatura es adecuada para los socios } \\
\text { comunitarios participantes }\end{array}$ & 32 & 86,5 \\
\hline $\begin{array}{l}\text { Creo que el socio comunitario tomará en cuenta las recomendaciones de mi } \\
\text { grupo de trabajo }\end{array}$ & 32 & 86,5 \\
\hline $\begin{array}{l}\text { El tipo de asesoría al socio comunitario es pertinente para cumplir los aprendi- } \\
\text { zajes de la asignatura }\end{array}$ & 30 & 81,1 \\
\hline $\begin{array}{l}\text { La relación entre mi equipo de trabajo y el socio comunitario fue adecuada para } \\
\text { el cumplimiento de los objetivos }\end{array}$ & 30 & 81,1 \\
\hline $\begin{array}{l}\text { En la asignatura hubo adecuadas instancias de reflexión en torno al desarrollo } \\
\text { de la actividad de vinculación con el medio }\end{array}$ & 28 & 75,7 \\
\hline $\begin{array}{l}\text { El tiempo estimado para planificar y elaborar el producto solicitado fue ade- } \\
\text { cuado }\end{array}$ & 28 & 75,7 \\
\hline $\begin{array}{l}\text { La actividad de vinculación con el medio tuvo una duración adecuada para } \\
\text { lograr los objetivos propuestos }\end{array}$ & 27 & 73,0 \\
\hline $\begin{array}{l}\text { Me gustaría desempeñarme laboralmente en trabajos similares a los de la acti- } \\
\text { vidad de vinculación desarrollada en esta asignatura }\end{array}$ & 23 & 62,2 \\
\hline Las calificaciones que obtuve en la asignatura reflejan mis aprendizajes & 22 & 59,5 \\
\hline
\end{tabular}


Como se observa en la tabla expuesta, la evaluación general de los estudiantes acerca de la metodología de vinculación con el medio es positiva. Los dos puntos de desacuerdo más importantes son los referidos a que las notas reflejan los aprendizajes adquiridos en la asignatura y la proyección de desempeñarse laboralmente en actividades como la realizada en la actividad de vinculación con el medio. Por otro lado, los puntos de acuerdo más importantes son aquellos referidos a que la actividad de vinculación con el medio es adecuada para el nivel de conocimientos, competencias y destrezas que tienen y que los aprendizajes del curso fueron significativos para ellos.

\section{COMPETENCIAS GENÉRICAS Y COMPORTAMIENTO CIUDADANO}

En cuanto a las competencias genéricas que los estudiantes consideraron que fueron trabajadas en mayor medida en el curso de Fundamentos de Costos con metodología $\mathrm{A}+\mathrm{S}$ fueron la competencia de trabajo en equipo $(89,2 \%)$, aprender de forma práctica $(62,2 \%)$ y responsabilidad y compromiso ciudadano (51,4\%). Estas tres son las competencias declaradas más reforzadas en la asignatura.

Tabla VII. Competencias Genéricas reforzadas por la asignatura.

\begin{tabular}{|l|c|c|}
\hline Competencias Genéricas & Frecuencia & $\%$ \\
\hline Trabajo en equipo & 33 & 89,2 \\
\hline Aprender de forma práctica & 23 & 62,2 \\
\hline Responsabilidad social y compromiso ciudadano & 19 & 51,4 \\
\hline Compartir conocimientos & 16 & 43,2 \\
\hline Rigurosidad Profesional & 14 & 37,8 \\
\hline Comunicación efectiva & 12 & 32,4 \\
\hline Mediación de conflictos & 10 & 27,0 \\
\hline Liderazgo & 9 & 24,3 \\
\hline Pensamiento crítico & 5 & 13,5 \\
\hline Cooperación ante la rivalidad & 5 & 13,5 \\
\hline Aceptación de la diferencia & 3 & 8,1 \\
\hline Adquisición de segunda lengua & 0 & 0,0 \\
\hline Discernimiento ético & 0 & 0,0 \\
\hline
\end{tabular}

Respecto del comportamiento cívico, se consultó a los estudiantes acerca de la frecuencia con que realizaban ciertas acciones sociales y políticas. De esa pregunta se extrajeron los siguientes resultados: 
Tabla VIII. Acciones de Comportamiento Cívico.

\begin{tabular}{|l|c|c|}
\hline \multicolumn{1}{|c|}{ Acciones de Comportamiento Cívico } & $\begin{array}{c}\% \text { Baja } \\
\text { frecuencia }\end{array}$ & $\begin{array}{c}\% \text { Alta } \\
\text { frecuencia }\end{array}$ \\
\hline Ayudo a los demás cuando me lo piden & 10,8 & 89,2 \\
\hline Gestos solidarios & 40,5 & 59,5 \\
\hline $\begin{array}{l}\text { Entiendo la información referida a cuestiones públicas (Procesos } \\
\text { de votación, consultas ciudadanas, etc.) }\end{array}$ & 40,5 & 59,5 \\
\hline $\begin{array}{l}\text { Hago uso de los mecanismos oficiales de participación política (por } \\
\text { ejemplo, elección de representantes políticos) }\end{array}$ & 43,2 & 56,8 \\
\hline Conozco y ejerzo mis derechos y deberes ciudadanos & 48,6 & 51,4 \\
\hline $\begin{array}{l}\text { Hago uso de los mecanismos oficiales de participación universita- } \\
\text { ria (por ejemplo, elección de representantes estudiantiles) }\end{array}$ & 51,4 & 48,6 \\
\hline Investigo sobre el sistema político y social de mi país & 64,9 & 35,1 \\
\hline Realizo acciones orientadas al bienestar de mi comunidad & 67,6 & 32,4 \\
\hline $\begin{array}{l}\text { Me organizo para dar solución a los problemas que aquejan mi co- } \\
\text { munidad cercana }\end{array}$ & 81,1 & 18,9 \\
\hline
\end{tabular}

Como se observa en la tabla, las acciones realizadas con mayor frecuencia son brindar ayuda a los demás cuando se lo piden y realizar gestos solidarios, por otro lado las acciones menos realizadas fueron organizarse para dar solución a los problemas que aquejan su comunidad cercana.
Se solicitó a los estudiantes que indicaran si es que participaban de organizaciones dentro de la universidad, fuera de la universidad y si es que habían realizado algún tipo de voluntariado durante el último año. Al respecto los resultados son los siguientes:

Tabla IX. Participación en organizaciones de la sociedad civil.

\begin{tabular}{|l|c|c|}
\hline Participación & Frecuencia & $\%$ \\
\hline Participación dentro de la universidad & 9 & 24,3 \\
\hline Participación fuera de la universidad & 10 & 27,0 \\
\hline Voluntariado & 10 & 27,0 \\
\hline
\end{tabular}

Como se puede observar en la tabla, poco menos de un cuarto de los estudiantes de Fundamento de costos participa dentro de la universidad, mientras que el $27 \%$ de los estudiantes participan fuera de la universidad, y la misma cantidad ha realizado voluntariado.

\section{CONCLUSIONES}

A nivel general, se puede concluir que la implementación del Aprendizaje y Servicio en cursos de pregrado permite no solo acercar al estudiante a los desafíos reales de los microempre- 
sarios y el ejercicio profesional, sino que además permite el desarrollo de competencias genéricas valoradas en el mercado laboral. Además, cabe destacar que la metodología permite alinear los propósitos de la asignatura con las motivaciones personales de los estudiantes, lo que se refleja en una alta satisfacción por parte del estudiantado respecto al Aprendizaje y Servicio en la asignatura de Fundamento de Costos.

En primer lugar, las motivaciones de los estudiantes se asocian a elementos más intrínsecos y de crecimiento personal que a motivaciones extrínsecas, como lo pudieran ser el reconocimiento por parte de otros o de la universidad. Lo anterior, plantea un desafío para las innovaciones que se quieran hacer en la formación, en tanto estas deben estar alineadas con las motivaciones intrínsecas de los estudiantes, más que la valoración que el docente o la institución pueda otorgarle a ciertas actividades o competencias.

Asimismo, destaca la alta motivación social de los estudiantes, que se infiere de la importancia que le otorgan los mismos a tener conciencia social y ser un profesional que aporte a los desafíos del país. En ese sentido, la implementación de la Metodología de Aprendizaje y Servicios estaría alineada con las motivaciones personales de los estudiantes de la Facultad de Economía y Negocios. Por otro lado, el A+S también les permitiría acercarse al mundo laboral, señalada como el principal objetivo por el cual los estudiantes cursan la asignatura.

Por otro lado, y en términos de la evaluación que hacen los estudiantes de la experiencia de Aprendizaje y Servicio vinculada a la asignatura de Fundamento de Costos, cabe destacar que la participación de todos los actores es bien evaluada, tanto en términos de relaciones interpersonales, compromiso y comunicación. Los elementos anteriores son centrales para poder realizar una implementación exitosa de la metodología, en la cual se cumplan las expectativas de los diversos actores involucrados. Lo anterior puede verse reflejado en la excelente evaluación que hacen los estudiantes respecto a la implementación y utilidad de la metodología para sus aprendizajes y futuro desempeño profesional.

Asimismo, los estudiantes destacan como las tres competencias genéricas mayormente trabajadas en la asignatura, el trabajo en equipo, el aprender de forma práctica y la responsabilidad social y compromiso ciudadano. Esta última, si bien concentra un porcentaje superior al $50 \%$, debiera ser más evidente para los estudiantes, considerando que es la competencia clave a de- sarrollar en el curso. En ese sentido, queda como desafío vincular desde la reflexión en aula con los estudiantes como la experiencia de trabajo con micro-empresarios también es una acción de responsabilidad social y genera impactos positivos en el medio.

Esta baja identificación con la responsabilidad social universitaria, pudiera estar afectando la evaluación que hacen los estudiantes respecto a su comportamiento cívico. En ese sentido, a pesar de haber estado un semestre completo involucrados en una experiencia de Aprendizaje y Servicio, consideran no involucrarse mayormente en los problemas que le aquejan a su comunidad cercana y mayoritariamente visualizan que otorgan ayuda a otros cuando se les solicita.

En términos de las futuras investigaciones queda pendiente el desarrollo de instrumentos que permitan evaluar el impacto del Aprendizaje y Servicio de manera directa, es decir, en los aprendizajes de los estudiantes. Aun así, se considera un aporte en términos de avanzar hacia la sistematización de resultados de la aplicación de la metodología de Aprendizaje y Servicio en las disciplinas asociadas a la economía y los negocios. Asimismo, sería interesante realizar un seguimiento para ver si el trabajo de asesoría de los estudiantes permite dejar capacidades instaladas en los microempresarios, como forma de asegurar el impacto en términos del servicio social.

\section{REFERENCIAS BIBLIOGRÁFICAS}

Bringle R. \& Hatcher J. (1995). Service Learning Curriculum for Faculty. Michigan Journal of Community Service-Learning. 2, 112.

Flannery, B \& Pragman, C. (2008) Working towards empirically-based continuos improvement in service learning. Journal of Business Ethics, Vol. 80 No3 pp. 465-479.

Furco, A. (1990). El rol del docente en el desarrollo de proyectos de aprendizaje-servicio. In 3er y 4to Seminario Internacional: Escuela y Comunidad: La propuesta pedagógica del aprendizaje-servicio (pp. 35-39). California: Universidad de Berkeley.

Furco, A. (1998). Impacto Educacional del Aprendizaje-servicio ¿Qué sabemos a partir de la investigación? California: Universidad de Berkeley.

Kenworthy, A. (1996) Linking Business Edu- 
cation, Campus Culture and Community: The Bentley Service-Learning Project. Journal of Business Ethics, Vol. $15 \mathrm{~N}^{\circ} 1$ pp. 121-131.

Puig, J., Beatlle, R., Bosch, C., \& Palos, J. (2007). Aprendizaje y servicio solidario. Educar para la ciudadanía. Barcelona: Editorial Octaedro.

Puig J. (2009). Aprendizaje Servicio. Educación y Compromiso Cívico. Barcelona: Graó.

Rama, D. V. (1998). Learning by doing: Concepts and models for service-learning in accounting. Stylus Publishing, LLC.

Tapia, M. N. (2010). Aprendizaje y servicio solidario. En el sistema educativo y las organizaciones juveniles. Ciudad Nueva: Buenos Aires.

Wittmer, D. (2004) Business and Community: Intergrating Service Learning in Graduate Business Education. Journal of Business Ethics, Vol. 51 No4 pp. 359-371.

Zabalza, M. (2002). La Enseñanza Universitaria. El escenario y sus protagonistas, Madrid: Narcea.

Zamora, V. (2012) Using a Social Enterprice Service-Learning Strategy in an Introductory Managment Accounting Course. Issues In Accounting Education. Vol. $27 \mathrm{~N}^{\circ} 1$.

Zlotkowski, E. (1996) Opportunity for all: linking service-learning and business education. Journal of Business Ethics, Vol. 15 No1 pp. 5-19. 